\title{
Acoustic Wave Sparsely-Activated Localization Microscopy (AWSALM): In Vivo Fast Ultrasound Super-Resolution Imaging using Nanodroplets
}

\author{
Ge Zhang ${ }^{1}$, Sevan Harput ${ }^{1,2}$, Matthieu Toulemonde ${ }^{1}$, Jiaqi Zhu' ${ }^{1}$, Kai Riemer ${ }^{1}$, Kirsten Christensen-Jeffries ${ }^{3}$, Jemma \\ Brown $^{3}$, Robert J. Eckersley ${ }^{3}$, Peter Weinberg ${ }^{1}$, Chris Dunsby ${ }^{4}$, Meng-Xing Tang ${ }^{1}$ \\ ${ }^{1}$ Department of Bioengineering, Imperial College London, London, UK, SW7 2AZ, United Kingdom \\ ${ }^{2}$ Division of Electrical and Electronic Engineering, London South Bank University, London, SE1 OAA, UK \\ ${ }^{3}$ Biomedical Engineering Department, School of Biomedical Engineering and Imaging Sciences, Kings College London, London, \\ SE1 7EH, UK \\ ${ }^{4}$ Department of Physics and Centre for Pathology, Imperial College London, London, SW7 2AZ, UK \\ * These authors contributed equally to this work \\ Email: Mengxing.Tang@Imperial.ac.uk
}

\begin{abstract}
Current localization-based super-resolution ultrasound imaging requires a low concentration of flowing microbubbles to visualize microvasculature beyond the diffraction limit and acquisition is slow. Nanodroplets offer a promising solution as they can be sparsely activated and deactivated on-demand. In this study, acoustic wave sparselyactivated localization microscopy (AWSALM) using activation and deactivation of nanodroplets, an acoustic counterpart of photo-activated localisation microscopy (PALM) which is less dependent on agent concentration and the presence of flow, is demonstrated for super-resolution imaging in deep tissues in vivo. An in vivo super-resolution image of a rabbit kidney is obtained in 1.1 seconds using AWSALM, where microvessels with apparent sizes far below the half-wavelength of $220 \mu \mathrm{m}$ were visualized. This preliminary result demonstrates the feasibility of applying AWSALM for in vivo super-resolution imaging. .
\end{abstract}

Keywords- Super-Resolution, Octafluoropropane Nanodroplets, High Frame Rate, Flow Independent, Microbubbles

\section{INTRODUCTION}

Ultrasound super-resolution imaging techniques have shown the capability of breaking the diffraction limit in spatial resolution [1-7]. However, these current localization-based ultrasound super-resolution imaging techniques rely on a low concentration of active flowing microbubble signals. This means that, for slow flows associated with the microvasculature, a longer acquisition time is required for the microbubbles to replenish. Therefore, there is a need to develop a super-resolution imaging technique to acquire images faster to facilitate the clinical translation.

Nanodroplets, also called 'phase-change contrast agents', have been widely investigated in the field of medical ultrasound imaging [8-10]. In contrast enhanced ultrasound imaging, there are three main advantages of using nanodroplets compared with the conventional microbubble contrast agents. First, the diameter of a nanodroplet is much smaller than a microbubble: previous theoretical work calculated that nanodroplets are 3-6 times smaller than microbubbles [11]. Their smaller size makes it possible for nanodroplets to flow into smaller vessels or extravasate into tumour tissue where microbubbles cannot. Second, the in vivo circulation time of nanodroplets has been shown to be at least 3-5 times longer than that of microbubbles [12]. This can potentially allow the scanning session to last longer and the nanodroplet dose to be smaller. Third, the nanodroplets can be manipulated/activated by an ultrasound pulse sequence at a specific time or spatial location to become microbubbles to provide contrast signals. This provides more control and flexibility to the real-time scanning.

The use of high and low boiling-point nanodroplets for super-resolution have been reported [13] [8]. The imaging of repeated activation and re-condensation of high-boiling-point perfluorohexane (boiling-point $=56^{\circ} \mathrm{C}$ ) nanodroplets can be used to generate super-resolution photoacoustic imaging. However, the optical activation of the high-boiling-point nanodroplets via laser pulses was required, which restricts its penetration depth to regions that can be illuminated optically.

An acoustic counter-part of optical localization microscopy $[14,15]$, which we called acoustic sparsely activated localization microscopy (AWSALM), has been proposed in our previous study which utilised low-boiling-point decafluorobutane (boiling-point $=-2^{\circ} \mathrm{C}$ ) nanodroplets. AWSALM is a flow-independent technique and it does not require a low concentration of contrast agents. Compared to high-boiling-point nanodroplets, low-boiling-point nanodroplets have a much lower activation threshold and can be activated by diagnostic ultrasound pulses at depth.

Previous studies have shown AWSALM can be used to achieve super-resolution in vitro in a crossed-tube phantom. The later developed fast-AWSALM has shown the feasibility to obtain the super-resolution on s sub-second scale. The aim of this study is to demonstrate the feasibility of applying AWSALM in vivo. 


\section{METHODS AND EXPERIMENT}

\section{A. Nanodroplet Preparation}

The preparation of the nanodroplet solution has been adapted from previously described methods [16, 17]. The lipid shell was generated by dissolving 1,2-dipalmitoyl-sn-glycero3-phosphocholine (DPPC) and 1,2-distearoyl-sn-glycero-3phosphoethanolamine-N-[amino(polyethylene glycol)-2000] (DSPE-PEG-2000) into a solution of phosphate-buffered saline (PBS), propylene glycol, and glycerol. All the lipids described above were purchased from Avanti Polar Lipids, Inc., USA. The lipid solution was added to a glass vial and the headspace of vial was exchanged with decafluorobutane gas (Fluoromed, USA) via an inlet needle along with a vent needle. Mechanical agitation was sufficient to produce the formation of lipid-shell octafluoropropane-core microbubbles. In order to condense microbubbles into nanodroplets, the headspace of the vial was pressurised while the vial immersed in the ice-water bath according to the previously described method [18].

\section{B. Animal Preparation}

A specific pathogen-free male New Zealand rabbit (HSDIF strain; Envigo, Hillcrest, United Kingdom) was used in this study and all animal experiments complied with the Animals (Scientific Procedures) Act 1986 and were approved by the local ethical review panel. The rabbit was aged 8 weeks and individually housed in pens at $18-22{ }^{\circ} \mathrm{C}$ on a 12-hour light period and was fed a standard laboratory diet.

\section{In Vivo Protocol}

Fentanyl and fluanisone $(0.3 \mathrm{~mL} / \mathrm{kg})$ and midazolam $(0.025$ $\mathrm{mL} / \mathrm{kg}$ ) were administered through the marginal vein of each rabbit to produce general anesthesia. Maintenance doses were given as required. The rabbit was placed on a warming pad to maintain body temperature at $37^{\circ} \mathrm{C}$ and artificially ventilated. A $0.4 \mathrm{~mL}$ intravenous bolus of concentrated decafluorobutane nanodroplets $\left(10^{\wedge} 10\right.$ droplets $\left./ \mathrm{mL}\right)$ were injected in the rabbit prior to ultrasound scanning of the rabbit kidney.

\section{Image Acquisition}

A customised 'Imaging - Activation - Imaging' sequence [19-21] was implemented on an ultrasound research platform (Verasonics Vantage 128, Kirkland, USA) with a L11-4 38$\mathrm{mm}$ linear array probe (ATL, USA). Focused pulses $(\mathrm{MI}=1.3$ ) were transmitted to activate the nanodroplets and interleaved with plane waves $(\mathrm{MI}=0.25$, frame rate $=1000 \mathrm{~Hz})$ for imaging with a transmit frequency of $3.5 \mathrm{MHz}$ (Diffraction limit: half wavelength at $3.5 \mathrm{MHz}$ is $220 \mu \mathrm{m}) .1000$ images were acquired over 1.1 seconds (1.0 s imaging time $+0.1 \mathrm{~s}$ activation time).

\section{E. Image Analysis}

Singular value decomposition (SVD) processing was used to separate the activated nanodroplet (i.e. microbubble) signal by removing the background. The SVD thresholds were automatically determined from the location of the largest gradient on the energy versus singular value order curve. After SVD processing, super-localization was performed to reject the noise and detect potential vaporized droplets as described in [8]. The location of single isolated vaporized droplets was calculated by the "centroid" method. The resulting superresolution map was created from all the localizations detected over all the imaging frames.

\section{RESULTS AND DISCUSSIONS}
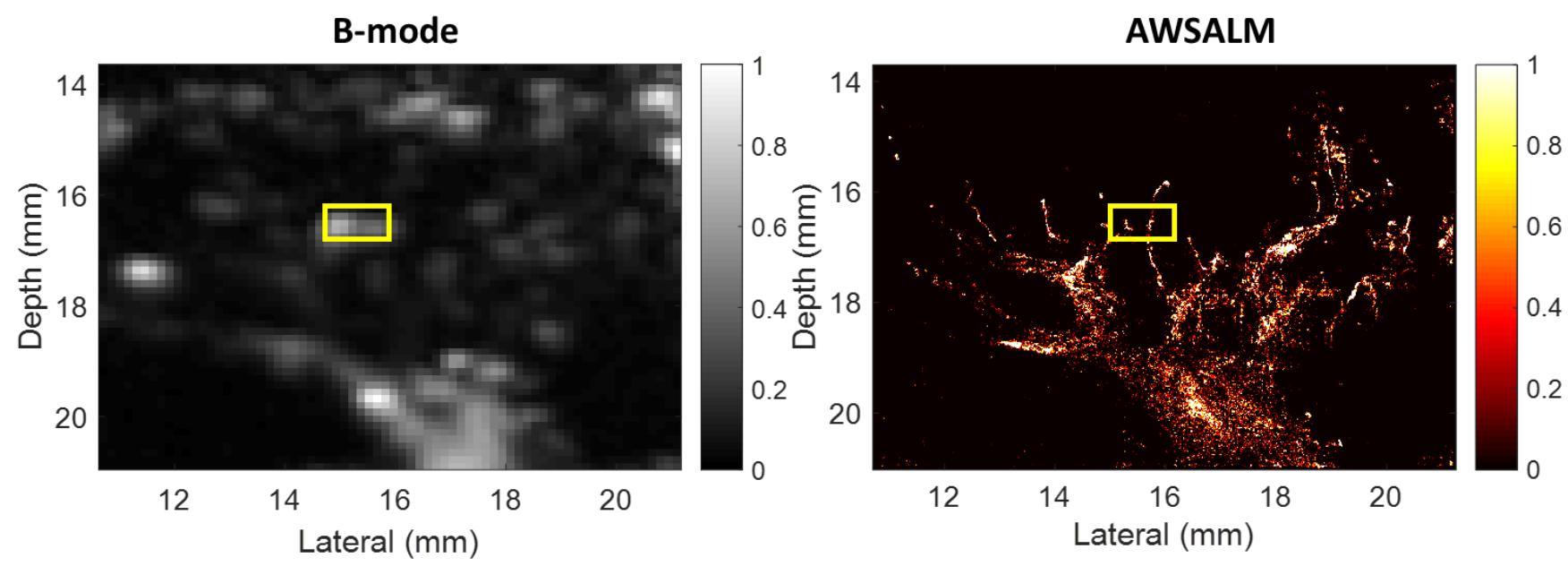

Fig. 1. (a) The summation of 1000 conventional B-mode image frames acquired in $1.1 \mathrm{~s}$ (b) Super-resolution image superimposes all the localization events in 1000 frames acquired in $1.1 \mathrm{~s}$. 
Fig 1 shows the comparison between the summation of 1000 conventional B-mode image frames acquired in $1.1 \mathrm{~s}$ and the corresponding super-resolution image which superimposes all the localization events in these 1000 frames. It can be seen that, in the super-resolution imaging, it resolves more microvasculature which cannot be visualized by B-mode image. A 40- $\mu \mathrm{m}$-diameter vessel can be visualized in the AWSALM image (which is much lower than the halfwavelength $220 \mu \mathrm{m}$ ) but not in the B-mode image.

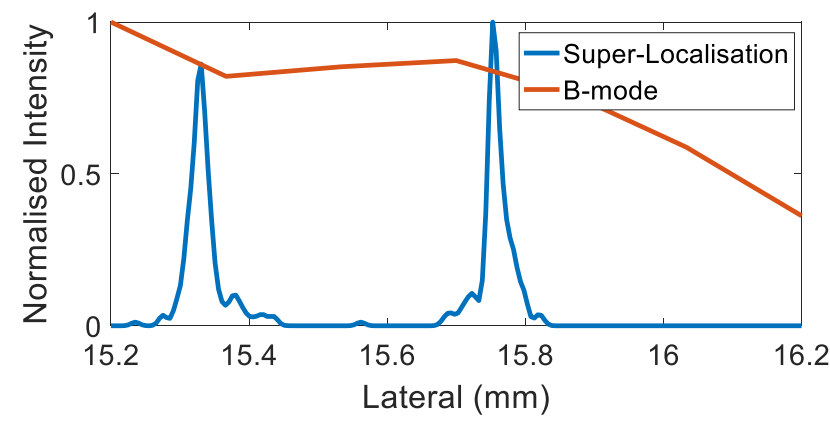

Fig. 2. Resolution measurement at the region of interest outlined by a yellow line in Fig. 1.

Fig 2 shows that, in the super-resolution imaging, two 40$\mu \mathrm{m}$-diameter vessels can be seen and the separation between two vessels is $420 \mu \mathrm{m}$. In the B-mode image, these two vessels cannot be visualized and quantified.

\section{CONCLUSION}

In summary, this study demonstrates AWSALM in a rabbit kidney. The result shows the in vivo super-localisation image can be obtained in 1.1 seconds using AWSALM. The AWSALM image resolves a more complex microvasculature network which cannot be resolved by B-mode imaging. Two vessels with apparent 40- $\mu$ m-diameter not resolvable in the Bmode image can be visualized in the AWSALM image. The acoustic pressure of the transmitted pulses was well within the FDA-approved safety range (mechanical index $<1.9$ ). It should be noted that AWSALM is also flow independent and does not require a precise control on contrast agent concentration, which opens the possibilities for super-resolution molecular imaging.

\section{ACKNOWLEDGMENT}

This work was supported by the EPSRC under Grant EP/N015487/1 and EP/N014855/1, in part by the King's College London (KCL) and Imperial College London EPSRC Centre for Doctoral Training in Medical Imaging (EP/L015226/1), in part by the Wellcome EPSRC Centre for Medical Engineering at KCL (WT 203148/Z/16/Z), in part by the Department of Health through the National Institute for Health Research comprehensive Biomedical Research Center Award to Guy's and St Thomas' NHS Foundation Trust in partnership with KCL and King's College Hospital NHS Foundation Trust, in part by the Graham-Dixon Foundation and in part by NVIDIA GPU grant. This work is also supported by the CRUK Multidisciplinary Project Award (No. C53470/A22353), and Imperial-ICR CRCE pump-priming awards.

\section{REFERENCES}

1. Viessmann, O.M., et al., Acoustic super-resolution with ultrasound and microbubbles. Phys Med Biol, 2013. 58(18): p. 6447-58.

2. Christensen-Jeffries, K., et al., 3-D In Vitro Acoustic Super-Resolution and Super-Resolved Velocity Mapping Using Microbubbles. IEEE Trans Ultrason, Ferroelectr, Freq Control, 2017. 64: p. 1478-1486.

3. Christensen-Jeffries, K., et al., In Vivo Acoustic Super-Resolution and Super-Resolved Velocity Mapping Using Microbubbles. Ieee Transactions on Medical Imaging, 2015. 34(2): p. 433-440.

4. Lin, F., et al., 3-D Ultrasound Localization Microscopy for Identifying Microvascular Morphology Features of Tumor Angiogenesis at a Resolution Beyond the Diffraction Limit of Conventional Ultrasound. Theranostics, 2017. 7(1): p. 196-204.

5. Errico, C., et al., Ultrafast ultrasound localization microscopy for deep super-resolution vascular imaging. Nature, 2015. 527(7579): p. 499-502.

6. Couture, O., et al., Ultrasound Localization Microscopy and SuperResolution: A State of the Art. IEEE Transactions on Ultrasonics, Ferroelectrics, and Frequency Control, 2018. 65(8): p. 1304-1320.

7. Song, P., et al., Improved Super-Resolution Ultrasound Microvessel Imaging with Spatiotemporal Nonlocal Means Filtering and Bipartite Graph-Based Microbubble Tracking. IEEE transactions on ultrasonics, ferroelectrics, and frequency control, 2018. 65(2): p. 149-167.

8. Zhang, G., et al., Acoustic wave sparsely activated localization microscopy (AWSALM): Super-resolution ultrasound imaging using acoustic activation and deactivation of nanodroplets. Applied Physics Letters, 2018. 113(1): p. 014101.

9. Zhang, G., et al. Investigation of Nanodroplet Adhesion to Endothelial Cells under Atheroprone Flow Conditions in 2018 IEEE International Ultrasonics Symposium (IUS). 2018.

10. Toulemonde, M., et al. Flow Visualization through Locally Activated Nanodroplets and High Frame Rate Imaging. in 2018 IEEE International Ultrasonics Symposium (IUS). 2018.

11. Sheeran, P.S., et al., Methods of Generating Sub-Micron Phase-Shift Perfluorocarbon Droplets for Applications in Medical Ultrasonography. IEEE Trans Ultrason Ferroelectr Freq Control, 2016.

12. Sheeran, P.S., et al., Contrast-enhanced ultrasound imaging and in vivo circulatory kinetics with low-boiling-point nanoscale phase-change perfluorocarbon agents. Ultrasound Med Biol, 2015. 41(3): p. 814-31.

13. Luke, G.P., A.S. Hannah, and S.Y. Emelianov, Super-Resolution Ultrasound Imaging in Vivo with Transient Laser-Activated Nanodroplets. Nano Lett, 2016. 16(4): p. 2556-9.

14. Dudok, B., et al., Cell-specific STORM super-resolution imaging reveals nanoscale organization of cannabinoid signaling. Nat Neurosci, 2015. 18(1): p. 75-86.

15. Betzig, E., et al., Imaging Intracellular Fluorescent Proteins at Nanometer Resolution. Science, 2006. 313: p. 1643-1645.

16. Lin, S., et al., Imaging of vaporised sub-micron phase change contrast agents with high frame rate ultrasound and optics. Physics in Medicine and Biology, 2018.

17. Lin, S., et al., Effects of microchannel confinement on acoustic vaporisation of ultrasound phase change contrast agents. Physics in Medicine \& Biology, 2017. 62(17): p. 6884.

18. Zhang, G., et al. Acoustic response of targeted nanodroplets postactivation using high frame rate imaging. in 2017 IEEE International Ultrasonics Symposium (IUS). 2017.

19. Lin, S., et al. Vaporising phase change ultrasound contrast agent in microvascular confinement. in 2016 IEEE International Ultrasonics Symposium (IUS). 2016.

20. Zhang, G., et al. Super-Localisation Ultrasound Imaging using Sparse Activation of Low-Boiling-Point Nanodroplets. in The 23rd European Symposium on Ultrasound Contrast Imaging. 2018. Rotterdam, Netherlands

21. Lin, S., et al. High frame rate ultrasound imaging of vaporised phase change contrast agents. in 2017 IEEE International Ultrasonics Symposium (IUS). 2017. 
